Measurement of the absolute gas gain and gain variations study in straw-tube detectors

This content has been downloaded from IOPscience. Please scroll down to see the full text. 2013 JINST 8 T08001

(http://iopscience.iop.org/1748-0221/8/08/T08001)

View the table of contents for this issue, or go to the journal homepage for more

Download details:

IP Address: 134.99.128.41

This content was downloaded on 13/12/2013 at 07:45

Please note that terms and conditions apply. 


\title{
Measurement of the absolute gas gain and gain variations study in straw-tube detectors
}

\section{P. Gianotti, ${ }^{a, 1}$ A. Kashchuk, ${ }^{b}$ O. Levitskaya, ${ }^{b}$ L. Passamonti, ${ }^{a}$ D. Pierluigi, ${ }^{a}$}

\section{A. Russo ${ }^{a}$ and M. Savriè}

\author{
${ }^{a}$ INFN Laboratori Nazionali di Frascati, \\ Frascati, Italy \\ ${ }^{b}$ Petersburg Nuclear Physics Institute, \\ Gatchina, St. Petersburg, Russia \\ ${ }^{c}$ INFN Sezione di Ferrara, \\ Ferrara, Italy \\ E-mail: paola.gianotti@lnf.infn.it
}

ABSTRACT: We present the results of the absolute gas gain measurement of a straw drift-tube filled with a binary gaseous mixture $\mathrm{Ar}-\mathrm{CO}_{2}(90-10)$ at 2 bar absolute pressure. The measurement has been performed using an intense $1.3 \mathrm{GBq}{ }^{137} \mathrm{Cs}$-source producing the primary ionization current. The results, as a function of the high voltage and gas parameters, were fitted and parameterized with a Diethorn's formula.

KEYWORDS: Wire chambers(MWPC, Thin-gap chambers, drift chambers, drift tubes, proportional chambers etc); Gaseous detectors; Counting gases and liquids

\footnotetext{
${ }^{1}$ Corresponding author.
} 


\section{Contents}

1 Introduction 1

2 Experimental setup 1

3 Measurement results 3

3.1 Primary ionization current 3

3.2 Gas gain 3

3.3 Straw tube wire diameter selection 4

4 Conclusions 5

\section{Introduction}

Straw trackers of various configurations have been built for many large scale experiments and presently are successfully taking data. The results show that these detectors exhibit high efficiency, good spatial resolution and that straw drift-tubes are reliable and attractive tracking devices, see $[1-3]$.

In order to optimize the design of a tracker for a given gas mixture, it is necessary to know the dependence of the gas gain as a function of the applied voltage and of the mixture working parameters. The literature reports many operating characteristics for various gas mixtures at atmospheric pressure [4], but few data exist above 1 bar gas pressure.

In the present article, we report our measurements of the straw tube gas gain and the systematic study of gas gain variation as a function of the applied voltage and gas pressure. The straw is filled with the $\mathrm{Ar}-\mathrm{CO}_{2}$ (90-10) mixture. The measured data have been fitted with a Diethorn's formula [8] to allow further studies of the gas gain variations as a function of the sense wire diameter.

This work has been performed to optimize the design and the performance of the PANDA straw tube trackers [6].

\section{Experimental setup}

The straw tubes that have been used for our measurements are produced by Lamina Dielectrics Ltd by winding and gluing two aluminized Mylar strips of thickness $12 \mu \mathrm{m}$ back-to-back (total straw thickness, including the glue, is $27 \mu \mathrm{m}$ ). The tube radius is $5 \mathrm{~mm}$, while that of the gold-plated $\mathrm{W}$-Re anode wire is $10 \mu \mathrm{m}$. Mylar has been preferred over Kapton because of its better mechanical properties, a higher Young's modulus and a superior tensile strength. The straw tubes have been filled with a two-component gas mixture $\mathrm{Ar}-\mathrm{CO}_{2}(90-10)$ at 1 bar overpressure, i.e. 2 bar absolute pressure. This overpressure stretches the straw tube and prevents the wire sagging. For a $0.75 \mathrm{~m}$ long straw, the chosen overpressure induces on the anode wire a tension equivalent to $40 \mathrm{~g}$. 


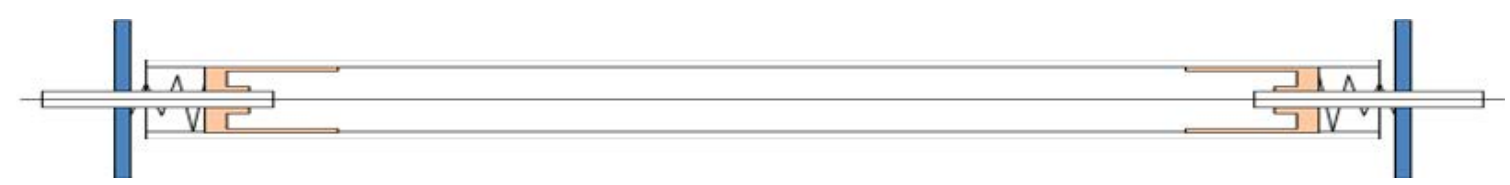

(a) Schematic cross-section of a single straw tube.

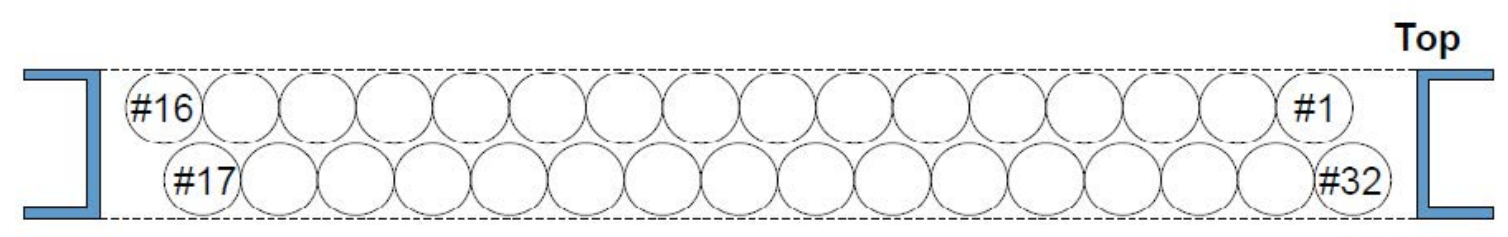

Bottom

(b) Frontal layout of the 32 straw tube module used for the measurements.

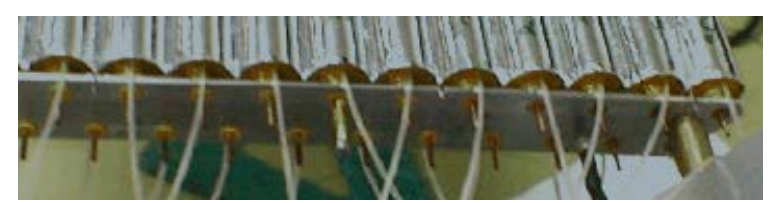

(c) Picture of the "far-end" of the straw tube module. Gas pipes for mixture supply are visible.

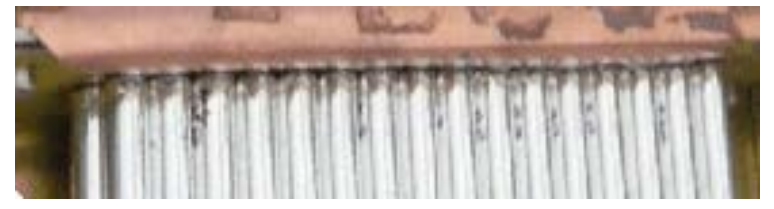

(d) Picture of the "near-end" of the straw tube module where the electronics is connected. The cathodes have been grounded with a Copper foil.

Figure 1: Details of the 32 straw tubes module used for the gas gain measurements.

We have built a module of 32 straw tubes, shown in figure 1, by fixing each straw to the neighboring with glue drops. This technique enhance the mechanical strength of the module that become self-supporting.

To determine the gas gain in the straw tubes, we have measured the primary ionization current produced when a very low voltage is applied to the anode-wire. The ionization is produced by the $662 \mathrm{keV} \gamma$-rays emitted by an intense $1.3 \mathrm{GBq}{ }^{137} \mathrm{Cs}$-source carefully screened and collimated by a lead case, see ref. [7]. The source can be moved along the whole module length as shown in figure 2. An auto-ranging Keithley-485 pico-ammeter with sensitivity $0.1 \mathrm{pA}$ has been connected between the straw cathode and the ground to measure the produced current. As shown in [7], the primary ionization current has to have the same value both for a low positive or negative voltage applied 


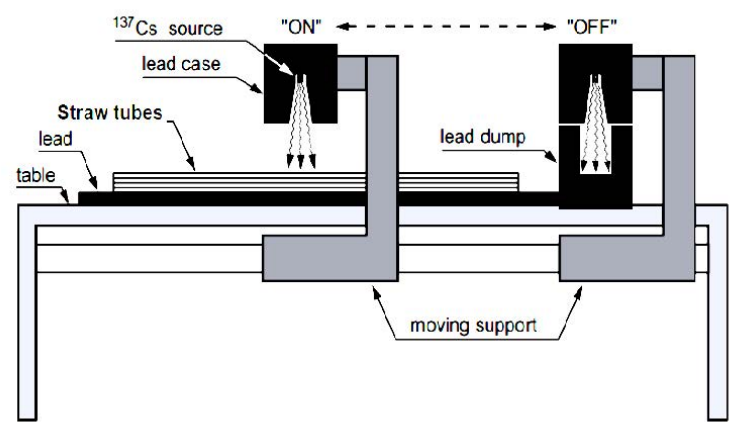

Figure 2: Experimental setup for the gas gain measurements with the ${ }^{137} \mathrm{Cs}$-source irradiating the straw tube module. The source can move over the full length of the straw tubes. When the source is in the "OFF" position it is completely screened by a lead case.

between the anode and the cathode. This value is constant over a wide range of voltages from 10 to $500 \mathrm{~V}$. To simplify our measurements, we used two blocks of batteries of $50 \mathrm{~V}$ and $100 \mathrm{~V}$ to set the voltage difference between the anode and the cathode. The batteries are an ideal floating power supply allowing accurate measurements of very small currents and avoiding parasitic ground loops.

\section{Measurement results}

\subsection{Primary ionization current}

The measurements of the primary ionization current have been averaged over 4 points: 2 points at $\pm 50 \mathrm{~V}$ and 2 at $\pm 100 \mathrm{~V}$. Two currents, $I^{+}$and $I^{-}$, have been recorded by switching the polarity of the batteries connected to the wire via a resistor of $10 \mathrm{M} \Omega$. Due to an offset of the Keithley-485, the measured currents do not change sign when the polarity of the batteries change (see figure 3 ). We have also measured the dark current when the source is in the "OFF" position, i.e. located out of the active area of the detector. The contribution of the dark current has been found negligibly small for both battery's blocks. The values of the ionization current measured at $\pm 50 \mathrm{~V}$ and $\pm 100 \mathrm{~V}$ are, as expected, very close. Therefore, we have determined the mean value of the primary ionization current averaging all our measurements: $I_{0}=1.70 \pm 0.21 \mathrm{pA}$. Similar results were obtained for different straws of the 32-straw module.

\subsection{Gas gain}

Once the primary ionization current $I_{0}$ has been determined, we have evaluated the gas gain $G$ at any voltage $V$, as the ratio $I / I_{0}$, where $I$ is the current corresponding to $V$. The voltage $V$ has been set in the range 1000-1900 $\mathrm{V}$.

By using a Diethorn's parameterization [8] we can write the gas gain $G$ as a function of the voltage, the density, and the detector geometry:

$$
G(V, \rho)=\frac{I}{I_{0}}=\left[\frac{V}{a \ln (b / a) \cdot E_{\min } \frac{\rho}{\rho_{0}}}\right]^{\frac{V}{\Delta V} \frac{\ln 2}{\ln (b / a)}}
$$




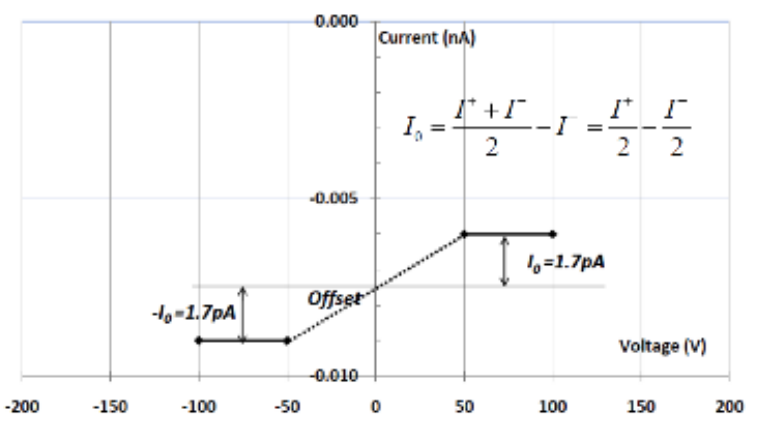

Figure 3: The figure illustrates how we have determined the primary ionization current. The measured currents $I^{+}$and $I^{-}$correspond to opposite battery polarities. These values are symmetric with respect to the pico-ammeter internal offset.

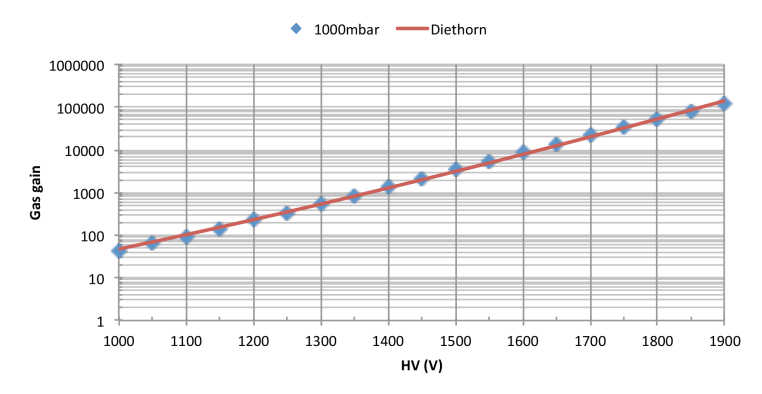

(a) Gas gain vs voltage for $\mathrm{Ar}-\mathrm{CO}_{2}(90-10)$ gas mixture at 2 bar absolute pressure (points) and fit performed with a Diethorn's function.

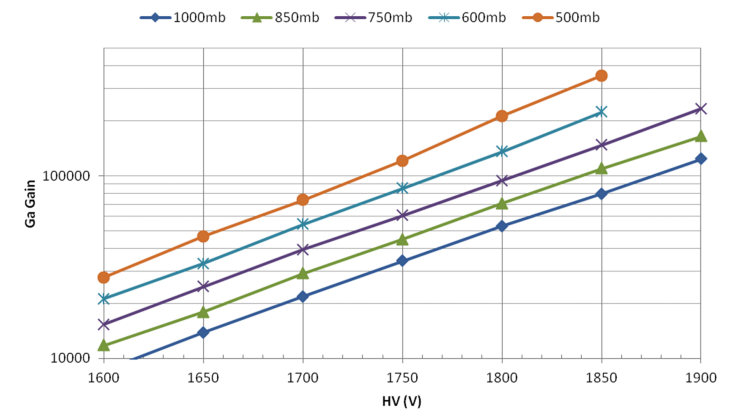

(b) Gas gain vs voltage at various overpressures. Points correspond to measurements, lines to fit functions.

Figure 4: Measured gas gain vs voltage.

here $a$ and $b$ are the radii of the anode wire and cathode tube, respectively. $E_{\min }$ is the minimal electric field needed for ionization, $\Delta V$ corresponds to the minimal voltage difference required to produce free electrons in an avalanche. $\rho / \rho_{0}=(P / T) /\left(P_{0} / T_{0}\right)$ is the ratio of gas density to the reference one with $P_{0}=1000$ mbar and $T_{0}=293 \mathrm{~K}$, respectively.

The results of the measurements are shown in figure $4 \mathrm{a}$ together with the result of a fit with a Diethorn's function with two free parameters: $E_{\min }$ and $\Delta V$.

We have also performed a set of measurements reducing the gas overpressure inside the straw tubes. In this way we checked that for a fixed HV value, the gas gain reduces as a function of the overpressure (figure $4 b$ ).

\subsection{Straw tube wire diameter selection}

The sense wire resistance, $R_{\text {wire }}$, is a key parameter for straw tubes performance. It determines the electric field value and therefore the avalanche structure. Furthermore, the dominant source of noise in a straw tube is not due to the used preamplifier, but to the wire resistance since noise r.m.s. depends on $\sqrt{R}_{\text {wire }}$. 


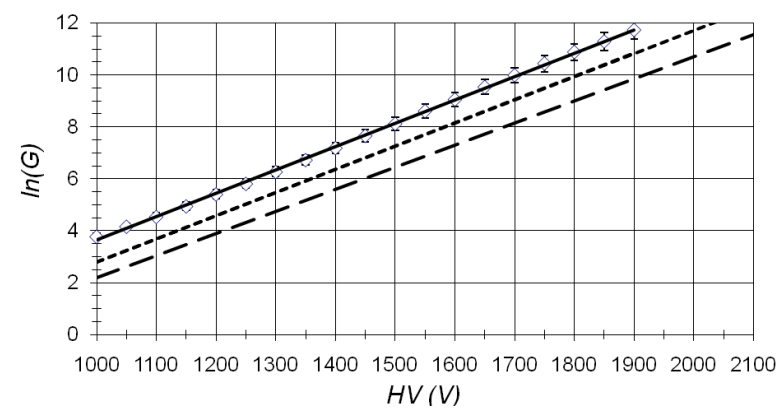

Figure 5: Gas gain measurements (points) with the best linear fit for the $20 \mu \mathrm{m}$ wire (solid line). Extrapolations of gas gain values for wire diameters $25 \mu \mathrm{m}$ and $30 \mu \mathrm{m}$ (dashed lines), obtained using the Diethorn's parameterization, are also shown.

The wire diameter we used in our test is $20 \mu \mathrm{m}$. The resistance of the wire, according to the data sheet of the company producing it is $R_{\text {wire }}=293 \Omega / \mathrm{m}$ [9]. If one takes a $30 \mu \mathrm{m}$ wire diameter, the resistance would be $R_{\text {wire }}=293 \times(20 / 30)^{2} \Omega / \mathrm{m}=130 \Omega / \mathrm{m}$, approximately a factor of 2 less than in the case of the $20 \mu$ m diameter wire and the noise r.m.s. will be reduced only by a factor $\sqrt{2}$.

In order to get a good spatial resolution with straw tubes it is important to have a large signalto-noise ratio. Therefore, it is important to increase the signal, increasing the gas gain, and to reduce the noise r.m.s. Nevertheless, the gas gain should not be too big since it can produce high space charge that can distort the resolution and prevents to run the detector at high rates.

We have then investigated how to get a gas gain of $\sim 10^{5}$ with a proper set of parameters using the Diethorn's parameterization obtained in the gas gain studies. We checked wire diameters of 20 , 25 and $30 \mu \mathrm{m}$ after having determined $E_{\min }$ and $\Delta V$ for our gas mixture. As is shown in figure 5 an increase of the wire diameter from $20 \mu \mathrm{m}$ implies that to get the same gas gain, the high voltage should be increased by $100 \mathrm{~V}(200 \mathrm{~V})$. This effect can be eventually used to reduce the r.m.s. noise level.

\section{Conclusions}

We performed gas gain measurements in a straw tube detector operated with a gas mixture Ar$\mathrm{CO}_{2}(90-10)$. We studied gas gain variations as a function of the applied high voltage at 2 bar absolute pressure, and we checked gas gain dependency from gas mixture pressure variations. The results have been fit with a Diethorn's function. In this way, we can predict gas gain changes due to modifications of the detector's operation parameters e.g. anode wire diameters and gas overpressure. The goal of this work was to chose the optimum working point of the PANDA straw tubes and to determine the characteristics of the detector signals.

This work has been partially supported by the EU project HadronPhysics3, Grant agreement no: 283286.

\section{References}

[1] LHCB OUTER TRACKER collaboration, Commissioning and performance of the LHCb outer tracker, Nucl. Instrum. Meth. A 628 (2011) 64. 
[2] V. Peshekhonov et al., Straw tube subsystem of the CBM muon detector, Part. Nucl. Lett. 9 (2012) 172

[3] ATLAS collaboration, Alignment of the ATLAS inner detector tracking system, Nucl. Instrum. Meth. A 623 (2010) 356.

[4] M. Deptuch, T.Z. Kowalski and B. Mindur, Gas gain in the straw tubes, Nucl. Phys. B 125 (2003) 385.

[8] W. Diethorn, A methane proportional counter system for natural radiocarbon measurements., US Atomic Energy Commission Report NYO-6628 (1956).

[6] $\bar{P} A N D A$ experiment, Antiproton Annihilation at Darmstadt, http://www-panda.gsi.de/.

[7] E. Dane, A. Sarti, G. Penso and D. Pinci, Detailed study of the gain of the MWPCs for the LHCb muon system, Nucl. Instrum. Meth. A 572 (2007) 682.

[8] W. Diethorn, A methane proportional counter system for natural radiocarbon measurements, US Atomic Energy Commission Report NYO-6628 (1956).

[9] LUMA Metall data sheet, http://www.luma-metall.se/assets/files/LUMA\%20Gold\%20Plated\%20tungsten\%20wire.pdf. 